\title{
Efeitos de 24 Semanas de Treinamento Resistido Sobre Índices da Aptidão Aeróbia de Mulheres Idosas
}

\section{Effects of $\mathbf{2 4}$ Weeks of Resistance Training on Aerobic Fitness Indexes of Older Women}

\author{
Marcelo Guido \\ Ricardo Moreno Lima',2 \\ Ronald Benford ${ }^{1}$ \\ Tailce Kaley Moura Leite ${ }^{1}$ \\ Rinaldo Wellerson Pereira ${ }^{1}$ \\ Ricardo Jacó de Oliveira' \\ 1. Programa de Pós-Graduação \\ Stricto Sensu em Educação Física, \\ Universidade Católica de Brasília, \\ Brasília, Brasil. \\ 2. Programa de Pós-Graduação \\ Stricto Sensu em Gerontologia, \\ Universidade Católica de Brasília, \\ Brasília, Brasil.
}

Endereço para correspondência: Universidade Católica de Brasília - UCB, Mestrado em Educação Física, QS 07, Lote 01, Prédio São João Bosco, Sala 119 - 71996-700 Taguatinga, DF - Brasil. E-mail: guidomss@yahoo.com.br

\begin{abstract}
RESUMO
Introdução e objetivo: O consumo de oxigênio pico ( $\mathrm{VO}_{2}$ pico) e o limiar anaeróbio (LA) são amplamente aceitos como importantes medidas da aptidão aeróbia. Tradicionalmente, o treinamento aeróbio é visto como o principal meio de se aumentar esses índices. Em contrapartida, o treinamento resistido (TR) não é tipicamente prescrito para este fim. Em indivíduos idosos, tem sido sugerido que o TR é capaz de aprimorar a capacidade aeróbia; entretanto, a temática é controversa. O objetivo do presente estudo foi verificar os efeitos do treinamento resistido sobre índices da capacidade aeróbia de mulheres idosas. Metodologia: Participaram voluntariamente 50 idosas, as quais foram distribuídas em dois possíveis grupos: grupo controle (GC $-n=$ 25; idade média 68,00 $\pm 6,38$ ) e grupo treinamento ( $G T-n=25$; idade média 68,04 $\pm 6,78$ anos). Todas as participantes realizaram teste ergoespirométrico em esteira conduzido até a exaustão voluntária antes e após a intervenção. O GT foi submetido a um programa de TR para os principais grupos musculares três vezes por semana durante um período de 24 semanas. Ao GC foi solicitado que mantivessem suas rotinas habituais. Split plot Anova foi utilizada para verificar possíveis diferenças intra e intergrupos. Resultados: Foi observado que o GT apresentou aumento significativo nas variáveis tempo de teste e $\mathrm{VO}_{2}$, tanto no momento do LA como no momento da exaustão. No GC nenhuma das variáveis dependentes sofreu alteração significativa. Conclusão: Conclui-se que 24 semanas de TR é capaz de promover melhora no desempenho durante teste de esforço cardiopulmonar em uma amostra de mulheres idosas. Futuros estudos serão importantes para elucidar os mecanismos responsáveis por tais adaptações.
\end{abstract}

Palavras-chave: envelhecimento, capacidade aeróbia, treinamento de força, sarcopenia.

\section{ABSTRACT}

Introduction and objectives: Peak oxygen uptake $\left(\mathrm{VO}_{2}\right.$ peak) and anaerobic threshold (AT) have been widely accepted as important predictors of aerobic fitness. Traditionally, aerobic training is viewed as the main way to increase these indices. Conversely, resistance training (RT) is not typically prescribed for this purpose. In elderly subjects, it has been suggested that RT may increase aerobic capacity; however, the literature is controversial. The purpose of the present study was to verify the effects of 24 weeks of RT on aerobic capacity indexes of elderly women. Methods: Fifty elderly women voluntarily took part in this study and were divided into two possible groups: control group ( $C G-n=25$; mean age of $68.00 \pm 6.38$ years) and training group ( $T G-n=25$; mean age of $68.04 \pm 6.78$ years). All volunteers underwent cardiopulmonary exercise test on treadmill until volitional exhaustion before and after the intervention period. A split plot ANOVA test was used to examine differences within and between groups. Results: It was observed that the TG exhibited significant increase in the variables time of test and oxygen uptake for both AT and exhaustion moments. The CG did not show any significant alterations for any of the dependent variables. Conclusions: It can be concluded that 24 weeks of RT are capable of promoting improvement in performance during a cardiopulmonary exercise test in a sample of elderly women. Further studies are necessary to elucidate the mechanisms responsible for these adaptations.

Keywords: aging, aerobic capacity, strength training, sarcopenia. 


\section{INTRODUÇÃO}

O envelhecimento do sistema cardiorrespiratório está associado à redução da capacidade aeróbia máxima, geralmente expressa pelo consumo pico de oxigênio $\left(\mathrm{VO}_{2} \text { pico }\right)^{(1)}$. Mesmo em indivíduos saudáveis e fisicamente ativos, ocorre declínio da aptidão aeróbia após os 40 anos de idade, que se torna mais acentuado a partir da sexta década de vida ${ }^{(2)}$. Esse declínio está relacionado com a diminuição do débito cardíaco máximo e da diferença arteriovenosa de oxigênio, que também diminuem com o envelhecimento ${ }^{(3)}$. A modulação da função cardíaca pelo sistema nervoso autônomo diminui com o avançar da idade, ocorrendo declínio na resposta à estimulação adrenérgica do coração senescente. Esse processo repercute em menor cronotropismo e inotropismo, fazendo com que o débito cardíaco máximo diminua. Por outro lado, a diminuição na diferença arteriovenosa pode estar, em parte, associada ao declínio na força e massa muscular, processo conhecido como sarcopenia. Estudos demonstram que a sarcopenia pode também contribuir para o declínio da aptidão aeróbia associado ao avançar da idade ${ }^{(4)}$. De fato, dados recentes do nosso laboratório confirmaram relação significativa entre força e massa muscular com a aptidão aeróbia de indivíduos idosos ${ }^{(5)}$.

O teste de esforço cardiopulmonar, procedimento também chamado de ergoespirometria, permite a determinação objetiva do $\mathrm{VO}_{2}$ pico. Vacanti et al. ${ }^{(6)}$ relataram que o procedimento é seguro e eficaz para avaliar as respostas cardiovasculares, mesmo em indivíduos muito idosos. Além da determinação do consumo de oxigênio, a ergoespirometria possibilita a identificação do limar anaeróbio $(L A)^{(7)}$, variável considerada como medida reprodutível da capacidade aeróbia( ${ }^{(8)}$. O LA tem sido amplamente utilizado para prescrição de intensidades de esforço, tanto no âmbito da reabilitação como nos esportes de alto rendimento ${ }^{(9)}$. Intensidades acima do LA estão relacionadas a aumento dos níveis de catecolaminas e elevada percepção subjetiva de esforço ${ }^{(10)}$. Em indivíduos idosos é possível que algumas atividades rotineiras representem intensidades acima do LA, portanto, serão realizadas com considerável grau de desconforto. 0 consumo de oxigênio associado ao LA apresentou relação com força e massa magra de idosos em estudo prévio ${ }^{(5)}$ e intervenções capazes de aprimorar essa variável são positivas para a população em questão.

Nesse sentido, é bem aceito que o exercício físico sistematizado constitui intervenção capaz de retardar o declínio da aptidão aeróbia que acompanha o envelhecimento. Indivíduos que envelhecem praticando atividades físicas apresentam menores declínios na aptidão aeróbia quando comparados aos fisicamente inativos ${ }^{(11)}$. Exercícios cíclicos, envolvendo grandes grupos musculares, são tradicionalmente vistos como meio efetivo para este fim ${ }^{(12,13)}$. Em contrapartida, os exercícios resistidos são vistos como meio para incrementos na massa e força musculares, atenuando a progressão da sarcopenia e repercutindo positivamente no desempenho funcional de idosos ${ }^{(14-17)}$. No que concerne a aumento de $\mathrm{VO}_{2}$ pico ou LA, entretanto, a eficácia do treinamento resistido (TR) não parece ser muito importante ${ }^{(18,19)}$. Todavia, em indivíduos idosos nos quais parte do declínio da aptidão aeróbia parece ser atribuído à sarcopenia ${ }^{(4,5)}$, é possível que um programa de TR beneficie, por exemplo, índices obtidos em teste de esforço cardiopulmonar.

De fato, estudos envolvendo jovens e pessoas de meia-idade não demonstraram melhorias significativas na capacidade aeróbia em decorrência do TR ${ }^{(20-22)}$. Em idosos, poucos estudos examinaram essa temática e os resultados não são consensuais. Estudando indivíduos de ambos os sexos e com idade compreendida entre 60 e 83 anos, Vincent et al. (23) encontraram significativo aumento no $\mathrm{VO}_{2}$ pico após seis meses de TR. Esses achados corroboram um estudo prévio de Hagerman et al.(24). Em contrapartida, Ades et al. ${ }^{(18)}$ não demonstraram alterações de $\mathrm{VO}_{2}$ pico após 12 semanas de TR, em um estudo cuja amostra foi composta por 24 indivíduos com idade igual ou superior a 65 anos. A controvérsia entre os estudos requer esclarecimentos futuros. Dessa forma, o presente estudo teve por objetivo verificar os efeitos do TR sobre índices da capacidade aeróbia de mulheres idosas. Para tal, foi implementado programa de treinamento de 24 semanas e variáveis relacionadas à aptidão aeróbia foram mensuradas por meio de teste de esforço cardiopulmonar. Um grupo controle também foi examinado. Com base em achados prévios do nosso grupo ${ }^{(5)}$ e de outros disponíveis na literatura ${ }^{(23,24)}$, nós levantamos a hipótese de que o programa de TR será capaz de alterar favoravelmente alguns índices da aptidão aeróbia de mulheres idosas.

\section{MÉTODOS}

\section{Amostra}

As participantes do presente estudo eram residentes no Distrito Federal e faziam parte de um projeto mais amplo desenvolvido na universidade. O recrutamento da amostra fora publicado anteriormente em detalhes ${ }^{(5)}$. Um total de 50 idosas fizeram parte da presente investigação, as quais foram distribuídas em dois grupos: grupo treinamento (GT - n $=25$, idade média de 68,04 $\pm 6,78$ anos) e grupo controle (GC $-n=25$, idade média de $68,00 \pm 6,38)$. Os critérios de exclusão adotados foram os seguintes: implante de prótese metálica, limitações musculoesqueléticas ou cardiovasculares que contraindicassem a realização de esforço físico, caminhar apenas com assistência. Ademais, as voluntárias deveriam estar há pelo menos seis meses sem praticar atividades físicas regulares.

O estudo foi aprovado pelo Comitê de Ética da Universidade Católica de Brasília, registrado sob o ofício número CEP/UCB 014/2007. Adicionalmente, todas as participantes assinaram o Termo de Consentimento Livre e Esclarecido, o qual continha os objetivos dos procedimentos a serem realizados, bem como os possíveis desconfortos, riscos e benefícios decorrentes do protocolo experimental.

\section{Avaliação da composição corporal}

A massa corporal foi mensurada por meio de uma balança digital da marca Filizola ${ }^{\circledR}$, com capacidade máxima de $150 \mathrm{~kg}$ e resolução de $50 \mathrm{~g}$. Um estadiômetro de parede foi utilizado para mensurar a estatura das participantes, sendo o resultado expresso em centímetros, e com resolução de 0,1cm. A partir destas medidas calculou-se o índice de massa corporal (IMC = massa morporal (em kg) / estatura² (em metros).

A composição corporal foi mensurada através da absortometria de raios-X de dupla energia (DXA) modelo DPX-IQ (Lunar Corporation, Madison, WI, EUA). O procedimento teve o intuito de melhor caracterizar a amostra e forneceu valores de massa livre de gordura total (MLG) e percentual de gordura.

\section{Avaliação da força muscular}

A força do quadríceps foi mensurada utilizando-se o dinamômetro isocinético Biodex System 3 (Biodex Medical Systems, New York, EUA). Antes do teste, as participantes foram submetidas a cinco minutos de aquecimento em cicloergômetro com baixa carga e velocidade confortável. Após explicação detalhada dos procedimentos da avaliação, as voluntárias foram cuidadosamente posicionadas no assento do equipamento. O eixo de rotação do braço do dinamômetro foi alinhado com o epicôndilo lateral do fêmur do membro dominante das voluntárias. 0 local da aplicação da força foi posicionado aproximadamente dois centímetros do maléolo medial. Cintos fixados com Velcro ${ }^{\circledR}$ foram utilizados no tronco, pelve e coxa para evitar eventuais movimentos compensatórios. Após familiarização com o equipamento, o protocolo consistiu de três séries de quatro contrações musculares com 30 segundos de intervalo entre as séries ${ }^{(25)}$. O valor registrado para as análises posteriores foi o maior pico de torque (PT) das três séries, o qual foi expresso em valores absolutos $(\mathrm{Nm})$ e relativos à massa corporal $(\mathrm{Nm} / \mathrm{kg})$. Às participantes foi solicitado que realizassem as contrações com o maior vigor possível e encorajamentos verbais foram oferecidos durante a mensuração. A calibração do equipamento era realizada de acordo com as instruções do fabricante no início das sessões de avaliação. 


\section{Teste de esforço cardiopulmonar}

As participantes realizaram teste de esforço em esteira rolante (modelo RT300 Pro, Moviment, Brasil) conduzido sob o protocolo de rampa previamente elaborado para que a exaustão ocorresse em aproximadamente 10 minutos. O mesmo protocolo foi seguido por todas participantes. Antes do teste, as voluntárias foram monitoradas por eletrocardiograma de repouso em 12 derivações (ECG Digital, Micromed, Brasil). Depois disso, tiveram instruções básicas relacionadas aos procedimentos e à utilização da escala de percepção subjetiva de esforço monitorada utilizando-se como instrumento a escala de Borg ${ }^{(26)}$. As voluntárias foram instruídas a realizar o teste até o momento em que se sentissem incapazes de continuar. Durante o esforço, o eletrocardiograma em três derivações era monitorado e fornecia os valores de frequência cardíaca. A pressão arterial foi medida continuamente utilizando um esfigmomanômetro de coluna de mercúrio em intervalos de três minutos, enquanto a percepção subjetiva de esforço foi coletada em intervalos de dois minutos e na exaustão. Durante todo o teste, os gases expirados foram medidos continuamente "respiração por respiração", utilizando o aparelho Cortex Metalyzer 3B (Cortex Biophysik, Alemanha). O volume e as concentrações de $\mathrm{O}_{2}$ e $\mathrm{CO}_{2}$ foram calibrados de acordo com as instruções do fabricante. Todos os procedimentos foram realizados sob supervisão médica e as voluntárias foram autorizadas a realizar o teste apoiando-se nas barras frontais de apoio da esteira ergométrica. $\mathrm{O} \mathrm{VO}_{2}$ pico foi considerado como a média dos últimos 30 segundos de exercício. As medições da aptidão aeróbia foram realizadas no Laboratório de Estudos em Educação Física e Saúde (LEEFS), que tem temperatura ambiente controlada e equipamentos e medicamentos necessários para prestar cuidados de urgência.

O limiar anaeróbio foi determinado como o ponto em que ocorreu perda da linearidade entre a produção de dióxido de carbono e o consumo de oxigênio, identificada quando a curva do equivalente ventilatório de oxigênio e a pressão parcial de oxigênio atingiam valores mínimos seguidos de aumento sistemático.

\section{Programa de treinamento resistido}

Antes do TR, para familiarização com os equipamentos e aprendizado da correta técnica de execução dos movimentos, as voluntárias do GT foram submetidas a período de adaptação com duração de três semanas. Os exercícios que compuseram o programa foram: leg press, puxada pela frente, extensão de joelhos, supino vertical, flexão de joelhos, abdução de quadril, abdução de ombros, abdominal, flexão plantar ortostática.

Após o período de adaptação, foram realizados testes de uma repetição máxima $(1-R M)^{(27)}$, com o propósito de identificar a carga de treinamento. $O$ programa teve duração de 24 semanas, realizados em três sessões semanais (segundas, quartas e sextas), totalizando 72 sessões. Nas quatro semanas iniciais, a intensidade foi de 60\% de 1-RM (12 repetições); nas quatro semanas subsequentes, a intensidade foi de $70 \%$ de 1-RM (10 repetições); e, nas semanas restantes, a intensidade foi de $80 \%$ de 1-RM (oito repetições). A cada quatro semanas era aplicado o teste de 1-RM para ajustes das cargas. O método de treinamento adotado foi o alternado por segmento e os intervalos entre as séries e entre os exercícios foram de aproximadamente um minuto. Às participantes foi orientado que não alterassem suas rotinas habituais nem ingressassem em outros programas de atividades físicas. Só fizeram parte das análises as participantes que completaram $75 \%$ das sessões.

\section{Tratamento estatístico}

Inicialmente foi realizada análise exploratória dos dados, na qual se avaliou casos faltosos (missing cases), valores extremos (outliers) univariados e normalidade das variáveis dependentes. Split plot Anova \{ $\{2 \mathrm{x}$ 2) (Tempo [pré e pós] * Grupo [controle e treinamento])\} para medidas repetidas foi utilizada para verificar os efeitos da intervenção. Nesse sentido, os valores intrassujeitos foram as variáveis dependentes e os fatores intersujeitos foram os grupos (GC ou GT). Os dados estão apre- sentados por meio da estatística descritiva utilizando os procedimentos de média e desvio padrão. O nível de significância adotado foi de $p \leq$ 0,05 e o software SPSS versão 15.0 foi utilizado para todas as análises.

\section{RESULTADOS}

\section{Caracterização da amostra}

Os dados descritivos da amostra estão apresentados na tabela 1, tanto para o grupo total como para o GT e o GC. Não foram encontradas diferenças significativas em nenhuma das variáveis no momento préintervenção quando comparados os grupos controle e treinamento.

Tabela 1. Características descritivas da amostra total estudada, bem como dividida em grupos (Treinamento e Controle).

\begin{tabular}{|c|c|c|c|}
\hline \multirow[b]{2}{*}{ Variável } & \multirow{2}{*}{$\begin{array}{c}\text { Total } \\
(n=50)\end{array}$} & \multicolumn{2}{|c|}{ Grupos } \\
\hline & & $\begin{array}{c}\text { GT } \\
(n=25)\end{array}$ & $\begin{array}{c}G C \\
(n=25)\end{array}$ \\
\hline Idade (anos) & $68,00 \pm 6,58$ & $68,04 \pm 6,78$ & $68,00 \pm 6,38$ \\
\hline Massa corporal (kg) & $63,13 \pm 10,93$ & $60,96 \pm 8,08$ & $65,40 \pm 13,06$ \\
\hline Estatura $(m)$ & $1,51 \pm 0,06$ & $1,51 \pm 0,06$ & $1,53 \pm 0,08$ \\
\hline$I M C$ & $27,54 \pm 4,30$ & $27,02 \pm 3,32$ & $28,09 \pm 5,15$ \\
\hline MLGT & $36,7 \pm 4,25$ & $35,72 \pm 4,17$ & $37,49 \pm 4,99$ \\
\hline MLGA & $13,98 \pm 1,90$ & $13,60 \pm 1,87$ & $14,85 \pm 2,89$ \\
\hline$\%$ Gord & $39,29 \pm 5,84$ & $39,45 \pm 5,67$ & $38,07 \pm 6,06$ \\
\hline $\mathrm{VO}_{2} \mathrm{LA}\left(\mathrm{ml}\right.$.kg.min $\left.{ }^{-1}\right)$ & $12,25 \pm 2,25$ & $12,21 \pm 2,19$ & $12,30 \pm 2,40$ \\
\hline $\mathrm{VO}_{2} \mathrm{LA}\left({\left.\mathrm{L} . \mathrm{min}^{-1}\right)}^{-1}\right.$ & $0,77 \pm 0,17$ & $0,74 \pm 0,15$ & $0,81 \pm 0,18$ \\
\hline Tempo no LA (seg) & $300,54 \pm 78,24$ & $301,00 \pm 72,39$ & $300,00 \pm 86,89$ \\
\hline $\mathrm{VO}_{2}$ pico $\left(\mathrm{ml} . \mathrm{kg} \cdot \mathrm{min}^{-1}\right)$ & $17,09 \pm 3,31$ & $16,60 \pm 3,38$ & $17,60 \pm 3,23$ \\
\hline $\mathrm{VO}_{2}$ pico $\left(\right.$ L. min $\left.{ }^{-1}\right)$ & $1,09 \pm 0,27$ & $1,02 \pm 0,22$ & $1,16 \pm 0,30$ \\
\hline Tempo de teste (seg) & $595 \pm 133,32$ & $583,88 \pm 136,22$ & $605,76 \pm 132,09$ \\
\hline PT absoluto (Nm) & $92,90 \pm 23,48$ & $88,65 \pm 21,49$ & $98,17 \pm 25,27$ \\
\hline PT relativo (Nm.kg) & $146,34 \pm 34,35$ & $144,71 \pm 28,79$ & $152,53 \pm 37,03$ \\
\hline FCmax (bpm) & $132,67 \pm 14,07$ & $135,15 \pm 15,42$ & $130,09 \pm 12,29$ \\
\hline Rmax & $1,03 \pm 0,08$ & $1,03 \pm 0,09$ & $1,04 \pm 0,07$ \\
\hline
\end{tabular}

$\overline{\mathrm{IMC}}=$ índice de massa corporal; $\mathrm{MLGT}=$ massa livre de gordura do corpo inteiro, $\mathrm{MLGA}=$ massa livre de gordura apendicular; $\mathrm{PT}=$ pico de torque; $\%$ Gord. = percentual de gordura; $\mathrm{LA}=$ limiar anaeróbio; $\mathrm{VO}_{2} \mathrm{pico}=$ consumo pico de oxigênio; FCmax = frequência cardíaca máxima; Rmax = coeficiente respiratório máximo.

\section{Efeitos do treinamento resistido}

O programa de treinamento implementado foi bem aceito pelas voluntárias e não houve relatos de lesões causadas pela intervenção. Os efeitos do TR sobre os componentes da composição corporal e força muscular estão descritos na tabela 2. Para a composição corporal, não foram encontradas alterações significativas no GT, entretanto, houve tendência de aumento da MLGT $(P=0,07)$ e da MLGA $(P=0,13)$ e redução do percentual de gordura $(P=0,07)$. No GC não foram encontradas alterações significativas em nenhuma das variáveis. Em relação à força muscular dos extensores de joelho, foram observadas melhoras significativas $(p \leq 0,05)$ tanto para o PT absoluto quanto para o PT relativo à massa corporal após os seis meses de treinamento. No GC não houve alterações significativas em nenhuma das variáveis de força muscular. Em adendo, a Anova revelou interação significativa entre grupo*tempo para as variáveis relacionadas à força do quadríceps.

A tabela 3 apresenta os dados referentes aos efeitos do treinamento resistido sobre variáveis da aptidão cardiorrespiratória. O GT apresentou meIhoras significativas $(p \leq 0,05)$ em todas as variáveis analisadas, ou seja, $\mathrm{VO}_{2}$ (absoluto e relativo) e tempo de teste, sendo esses achados verdadeiros tanto no momento do LA quanto no momento da exaustão. A FC atingida no momento da exaustão (FCmax) também aumentou significativamente no GT. No GC não foram observadas alterações significativas para nenhuma das variáveis. Adicionalmente, foi notado que o tempo de teste associado ao LA após a intervenção foi maior no GT quando comparado ao GC. 
Tabela 2. Efeitos do treinamento resistido sobre componentes da composição corporal e força muscular.

\begin{tabular}{|c|c|c|c|c|c|c|}
\hline \multirow{2}{*}{ Variáveis } & \multicolumn{3}{|c|}{ GT } & \multicolumn{3}{|c|}{ GC } \\
\hline & Pré & Delta \% & Pós & Pré & Delta \% & Pós \\
\hline MLGT & $35,72 \pm 4,17$ & 2,21 & $36,51 \pm 3,91$ & $37,49 \pm 4,99$ & 1,39 & $38,01 \pm 4,95$ \\
\hline MLGA & $13,60 \pm 1,87$ & 3,82 & $14,12 \pm 2,00$ & $14,85 \pm 2,89$ & $-8,28$ & $13,62 \pm 4,06$ \\
\hline$\%$ Gord. & $39,45 \pm 5,67$ & $-2,69$ & $38,39 \pm 5,26$ & $38,07 \pm 6,06$ & $-2,76$ & $37,02 \pm 5,76$ \\
\hline PT absoluto (Nm) & $88,65 \pm 21,49$ & 18,65 & $105,18 \pm 23,98^{*}$ & $98,17 \pm 25,27$ & 2,00 & $100,13 \pm 20,94$ \\
\hline PT relativo (Nm.kg) & $1,45 \pm 0,28$ & 14,81 & $1,66 \pm 0,30^{*}$ & $1,52 \pm 0,37$ & 3,50 & $1,57 \pm 0,36$ \\
\hline
\end{tabular}

* Diferença significativa em relação aos valores pré intervenção $(p \leq 0,05)$.

$\mathrm{GT}=$ grupo treinamento; $\mathrm{GC}=$ grupo controle; $\mathrm{MLGT}$ = massa livre de gordura do corpo inteiro; $\mathrm{MLGA}=$ massa livre de gordura apendicular; \% Gord. = percentual de gordura; $\mathrm{PT}=$ pico de torque.

Tabela 3. Variáveis obtidas no teste de esforço cardiopulmonar antes e após os seis meses de intervenção.

\begin{tabular}{|c|c|c|c|c|c|c|}
\hline \multirow{2}{*}{ Variáveis } & \multicolumn{3}{|c|}{ GT } & \multicolumn{3}{|c|}{$\mathrm{GC}$} \\
\hline & Pré & Delta \% & Pós & Pré & Delta \% & Pós \\
\hline $\mathrm{VO}_{2} \mathrm{LA}\left(\mathrm{ml}\right.$.kg.min $\left.{ }^{-1}\right)$ & $12,21 \pm 2,19$ & 11,96 & $13,67 \pm 2,67^{*}$ & $12,30 \pm 2,40$ & 2,60 & $12,62 \pm 2,47$ \\
\hline $\mathrm{VO}_{2} \mathrm{LA}\left(\mathrm{L} \cdot \mathrm{min}^{-1}\right)$ & $0,74 \pm 0,15$ & 10,81 & $0,82 \pm 0,18^{*}$ & $0,81 \pm 0,18$ & 3,70 & $0,84 \pm 0,21$ \\
\hline Tempo no LA (seg.) & $301,00 \pm 72,39$ & 31,56 & $396,00 \pm 80,94^{*: \#}$ & $300,00 \pm 86,89$ & 6,27 & $318,82 \pm 91,23$ \\
\hline $\mathrm{VO}_{2}$ pico $\left(\mathrm{ml} . \mathrm{kg} \cdot \mathrm{min}^{-1}\right)$ & $16,60 \pm 3,38$ & 10,72 & $18,38 \pm 4,06^{*}$ & $17,60 \pm 3,23$ & 3,52 & $18,22 \pm 3,15$ \\
\hline $\mathrm{VO}_{2}$ pico $\left(1 . \mathrm{min}^{-1}\right)$ & $1,02 \pm 0,22$ & 10,78 & $1,13 \pm 0,24^{*}$ & $1,16 \pm 0,3$ & 4,31 & $1,21 \pm 0,30$ \\
\hline Tempo de teste (seg) & $583,88 \pm 136,22$ & 6,88 & $624,08 \pm 112,52^{*}$ & $605,76 \pm 132,09$ & $-1,57$ & $596,24 \pm 158,45$ \\
\hline FCmax (BPM) & $135,15 \pm 15,42$ & 9,25 & $146,38 \pm 14,39^{*}$ & $130,09 \pm 12,29$ & $-0,33$ & $129,47 \pm 16,05$ \\
\hline
\end{tabular}

* Diferença significativa $(P \leq 0,05)$ em relação aos valores pré intervençăo.

* Diferença significativa $(P \leq 0,05)$ em relação aos valores pós intervençăo do $G C$.

$\mathrm{LA}=$ limiar anaeróbio; $\mathrm{FC}$ max = frequência cardíaca máxima.

\section{DISCUSSÃO}

O presente estudo teve por objetivo verificar os efeitos do TR sobre índices da capacidade aeróbia de mulheres idosas (idade média de 68,00 $\pm 6,58$ anos). Para tal, foi implementado programa de seis meses de treinamento, em frequência semanal de três sessões e com intensidade progressiva respeitando-se a interdependência volume $\mathrm{x}$ intensidade. As variáveis relacionadas à aptidão aeróbia foram mensuradas por meio de teste de esforço cardiopulmonar em esteira rolante conduzido até a exaustão voluntária. Confirmando a hipótese apresentada, o programa de TR induziu aumentos significativos nos índices da aptidão aeróbia dessa população. Mais especificamente, as voluntárias submetidas ao treinamento foram capazes de permanecer por mais tempo em exercício e de atingir maior $\mathrm{VO}_{2}$ pico após a intervenção, enquanto que o grupo controle não apresentou alterações desses índices. Em adendo, o tempo de teste e consumo de oxigênio no momento do LA foram maiores após o treinamento quando comparados com a linha de base do estudo, sendo que esses achados não foram observados no grupo controle.

Os resultados observados estão de acordo com alguns estudos disponíveis na literatura; entretanto, a temática é ainda controversa. Frontera et al. ${ }^{(28)}$, após 12 semanas de condicionamento de força com exercícios para os extensores e flexores de joelho realizados a 80\% de 1-RM, em amostra composta por indivíduos saudáveis com idade compreendida entre 60 e 72 anos, relataram aumento significativo de $\mathrm{VO}_{2}$ max. Nesse mesmo sentido, Vincent et al. ${ }^{(23)}$, em um estudo envolvendo 62 indivíduos de ambos os sexos e com idade variando entre 60 e 83 anos, encontraram significativo incremento no $\mathrm{VO}_{2}$ pico em decorrência de seis meses de TR. De forma interessante, mesmo empregando métodos de avaliação e de treinamento diferentes, os estudos citados são consistentes com os presentes resultados. Por exemplo, Vincent et al. ${ }^{(23)}$ utilizaram um cicloergômetro para mensuração da aptidão aeróbia e Frontera et al. ${ }^{(28)}$ implementaram um programa de treinamento que contemplou apenas os membros inferiores. Outros estudos sinalizaram resultados similares, mas avaliaram a aptidão aeróbia por métodos indiretos ${ }^{(14-29)}$. Em contrapartida, Ades et al. ${ }^{(18)}$, em indivíduos com idade igual ou superior a 65 anos, relataram que após 12 semanas de programa de treinamento de força não foi observada alteração no consumo máximo de oxigênio.
Maiorana et al. ${ }^{(30)}$ verificaram os efeitos de 10 semanas de treinamento de força em circuito em 26 homens com idade média de $60 \pm$ 8,5 anos, e não encontraram alterações significativas no $\mathrm{VO}_{2}$ pico após o período de intervenção. A controvérsia entre os estudos pode estar relacionada a diferenças nos métodos de avaliação e de treinamento. No estudo de Ades et al. ${ }^{(18)}$ não foram detalhados os critérios de mensuração do $\mathrm{VO}_{2}$ max, e a relação volume $\mathrm{x}$ intensidade do treinamento pareceu desequilibrada no início da intervenção. O estudo de Maiorana et al. ${ }^{(30)}$ empregou 10 semanas de treinamento, tempo que talvez não seja suficiente para promover as alterações observadas no presente estudo.

Enquanto alguns estudos foram delineados com o intuito de examinar os efeitos do TR sobre a capacidade aeróbia máxima (i.e. $\mathrm{VO}_{2}$ pico; $V_{2}$ máx), poucos se preocuparam em verificar os efeitos sobre o LA. Essa análise é relevante, haja vista que o LA tem sido amplamente utilizado para prescrição de intensidades em programas de condicionamento físico ${ }^{(9)}$. Ademais, em indivíduos idosos, é possível que algumas atividades rotineiras representem intensidades acima desse marcador de intensidade. Um estudo realizado por Marcinik et al.(22), no qual a amostra estudada foi composta por 18 homens jovens (idade média de 29 anos), os resultados obtidos demonstraram aumento médio de 12\% no limiar de lactato como efeito de 12 semanas de TR. Entretanto, em indivíduos idosos, não foram identificados estudos que investigassem a temática. Os presentes resultados sugerem que o TR é capaz de aprimorar o LA de indivíduos idosos. Embora replicação desses resultados seja necessária em futuros estudos, os achados sugerem importante benefício do TR para essa população. O aumento do LA pode repercutir na realização de determinadas atividades físicas habituais em menor grau de desconforto. Esses achados podem explicar, pelo menos em parte, as observações de Hunter et al. ${ }^{(31)}$, os quais demonstraram que programa de 26 semanas de TR induziu aumento significativo do gasto energético com atividades físicas diárias em idosos, ou seja, os voluntários passaram a se movimentar mais. Embora o mecanismo seja especulativo, é possível que os voluntários do citado estudo ${ }^{(31)}$ tenham aumentado o LA e passaram a realizar mais atividade física ao passo que se sentiam mais confortáveis para tal. Outro fato importante é o de que o trabalho cardíaco aumenta desproporcionalmente em intensidades acima do $L A^{(32)}$, portanto, aumento do 
ponto de transição aeróbio-anaeróbio confere fator de proteção para eventos cardiovasculares agudos. De fato, McCartney et al. ${ }^{(33)}$ relataram que, para uma mesma intensidade absoluta, a resposta cardiovascular de idosos encontra-se atenuada após 12 semanas de TR.

Embora evidências demonstrem a possibilidade de o TR alterar favoravelmente índices da aptidão aeróbia de indivíduos idosos, os mecanismos responsáveis por essas adaptações não são totalmente conhecidos. É possível que, durante um teste de esforço, o idoso não consiga atingir seu limite cardiovascular devido a fadiga muscular precoce ${ }^{(23)}$. Shephard ${ }^{(34)}$ relata que os indivíduos mais idosos apresentam dificuldade em alcançar o $\mathrm{VO}_{2}$ max por conta de fraqueza muscular. Dessa forma, é possível que os aumentos de força muscular observados no presente estudo tenham propiciado aos idosos melhor desempenho durante o teste de esforço cardiopulmonar, fazendo com que eles explorassem mais o sistema cardiovascular. De fato, foi evidenciado no presente estudo que o grupo submetido ao treinamento atingiu FCmax média significativamente superior após a intervenção, achados que não foram observados no grupo controle. De forma não exclusiva é também plausível que o aumento da capacidade aeróbia das voluntárias que se submeteram ao TR seja, em parte, explicado pelo aumento da capacidade oxidativa muscular. Nessa direção, Frontera et al.(28) relataram que o incremento de $\mathrm{VO}_{2}$ max, decorrente de um programa de TR, foi acompanhado de aumento na densidade capilar do músculo, bem como da atividade de uma importante enzima do metabolismo oxidativo, a citrato sintase. Ademais, Hagerman et al. (24) atribuíram o aprimoramento do $\mathrm{VO}_{2}$ max a aumento proporcional das fibras tipo lla quando comparadas com as fibras musculares do tipo Ilb. Por outro lado, é pouco provável que adaptações centrais como aumento da câmara cardíaca e, consequentemente, aumento no volume sistólico, ocorram em decorrência do TR, adaptações essas que explicariam uma melhor aptidão aeróbia. Nesse sentido, Hagerman et al. ${ }^{(24)}$ observaram que, embora um programa de treinamento de força tenha melhorado o desempenho de idosos frente a teste ergométrico

\section{REFERÊNCIAS}

1. Hollenberg M, Yang J, Haight TJ, Tager IB. Longitudinal changes in aerobic capacity: implications for concepts of aging. Journal of Gerontology 2006;61:851-8.

2. Fleg JL, Morrell CH, Bos AG, Brant LJ, Talbot LA, Wright JG, et al. Accelerated longitudinal decline of aerobic capacity in healthy older adults. Circulation - Journal of the American Heart Association 2005;112:674-82

3. Weiss EP, Spina RJ, Holloszy JO, Ehsani AA. Gender differences in the decline in aerobic capacity and its physiological determinants during the later decades of life. Journal of Applied Physiology 2006;101:938-44

4. Fleg JL, Lakatta EG. Role of muscle loss in the age related associated reduction in VO2max. J Appl Physiol 1988;65:1147-51.

5. Oliveira RJ, Mota AM, Pitanga F, Bottaro M, Guido M, Leite TKM, et al. Association Between Sarcopeniarelated Phenotypes with Aerobic Capacity indexes of Older Women. Journal of Sports Science and Medicine (in press)

6. Vacanti LJ, Sespedes LB, Sarpi MO. Exercise stress testing is useful, safe, and efficient even in patients aged 75 years or older. Arq Bras Cardiol 2004;82:147-50

7. Wasserman K, Mcilroy MB. Detecting the threshold of anaerobic metabolism in cardiac patients during exercise. Am J Cardiol 1964;14:844-52

8. Yamamoto Y, Miyashita M, Hughson RL, Tamura S, Shinohara M, Mutoh Y. The ventilatory threshold gives maximal lactate steady state. Eur J Appl Physiol Occup Physiol 1991;63:55-9.

9. Meyer T, Lucia A, Earnest CP, Kindermann W. A conceptual framework for performance diagnosis and training prescription from submaximal gas exchange parameters-theory and application. International Journal of Sports Medicine 2005;26:38-48

10. Yazbek P Jr, Carvalho RT, Sabbag, LMS, Battistella LR. Ergoespirometria. Teste de esforço cardiopulmonar metodologia e interpretação. Arq Bras Cardiol 1998;71.

11. Ades PA, Toth MJ. Accelerated decline of aerobic fitness with healthy aging. What is the good news? Circulation - Journal of the American Heart Association 2005;112:624-6.

12. Poehlman ET, Goran MI, Gardner AW, Ades PA, Arciero PJ, Katzmanrooks SM, et al. Determinants of decline in resting metabolic rate in aging females. American Journal of Physiology - Endocrinology and Metabolism 1993;264:450-5.

13. Malbut KE, Dinan S, Young A. Aerobic Training in the 'oldest old': the effect of 24 weeks of training British Geriatrics Society 2002;31:255-60.

14. Assumpção CO, Prestes J, Leite RD, Urtado CB, Neto JB, Pellegrinotti IL. Efeito do treinamento de força periodizado sobre a composição corporal e aptidão física em mulheres idosas. Revista da Educaçãa Física/UEM 2008;19:581-90

15. Hakkinen K, Kraemer WJ, Pakarinrn A, Triplettmcbride T, Bride JM, Hakkinen A, et al. Effects of heavy resistance/power training on maximal strength, muscle morphology, and hormonal response patterns in 60-75 year-old men and women. Can J Appl Physiol 2002;27:213-31.

16. Sullivan DH, Wall PT, Bariola JR, Bopp MM, Frost MY. Progressive resistance muscle strength training on hospitalized frail elderly. Am J Phys Med Rehabil. 2001;80:503-9.

17. Frontera WR, Meredith CN, O'Reilly KP, Knuttgen HG, Evans WJ. Strength conditioning in older men Skeletal muscle hypertrophy and improved function. J Appl Physiol 1988;64:1038-44. sob o protocolo de Bruce, nenhuma alteração de estrutura ou dimensão cardíaca foi notada ao fazer exame ecocardiográfico.

De forma geral, os presentes resultados reforçam recomendações disponíveis na literatura acerca da inclusão do TR em programas de condicionamento físico para indivíduos com idade avançada. Consistente com estudos prévios ${ }^{(23,24,28,31)}$, o programa de treinamento implementado foi bem aceito pelas voluntárias e não houve relatos de lesões causadas pela intervenção. Não obstante, o TR deve, preferencialmente, complementar e não substituir o treinamento aeróbio, até porque os ganhos observados são modestos quando comparados aos induzidos por treinamento mais específico. Nesse sentido, é válido citar o estudo de Izquierdo et al. ${ }^{(35)}$ o qual demonstrou que, em indivíduos idosos, as adaptações específicas de cada tipo de treinamento não são negativamente afetadas quando os treinamentos são aplicados de forma combinada.

\section{CONCLUSÃO}

Com base nos resultados observados, é possível concluir que programa de TR relativamente longo (24 semanas) é capaz de alterar positivamente índices da aptidão aeróbia de mulheres idosas, avaliados por meio de teste de esforço cardiopulmonar. Mais especificamente, as melhoras foram observadas para o tempo de teste e consumo de oxigênio no momento da exaustão e no momento do LA, e constituem adaptações benéficas para essa população. Futuros estudos são necessários para elucidar os mecanismos responsáveis pelos resultados encontrados.

\section{AGRADECIMENTOS}

O presente estudo contou com o apoio da Coordenação de Aperfeiçoamento de Pessoal de Nível Superior (Capes), do Conselho Nacional de Desenvolvimento Científico e Tecnológico (CNPq) e da Universidade Católica de Brasília (UCB).

Todos os autores declararam não haver qualquer potencial conflito de interesses referente a este artigo.

18. Ades PA, Ballor DL, Ashikaga T, Utton JI, Nair KS. Weight training improves walking endurance in healthy eldery persons. Annals of Internal Medicine 1996;124:568-72.

19. Parker ND, Hunter GR, Treuth MS, Kekes-Szabo T, Kell SH, Weinsier R, et al. Effects of strength training on cardiovascular responses during a submaximal walk and a weight-loaded walking test in older females. Journal of Cardiopulmonary and Rehabilitation 1996;16:56-62.

20. Fahey TD, Brown CH. The effects of an anabolic steroid on the strength, body composition, and endurance of college males when accompanied by a weight training program. Med Sci Sports Exerc. 1973;5:272-6

21. Hickson RC, Rosenkotter MA, Brown MM. Strength training effects on aerobic power and short term endurance. Med Sci Sports Exerc 1980;12:336-9.

22. Marcinik EJ, Potts J, Schlabach G, Will S, Dawson P, Hurley BF. Effects of strength training on lactate threshold and endurance performance. Med Sci Sports Exerc 1991;23:739-43.

23. Vincent KR, Braith RW, Feldman RA, Kallas HE, Lowenthal DT. Improved Cardiorespiratory Endurance Following 6 Months of Resistance Exercise in Elderly Men and Women. Arch Intern Med 2002;162:673-8.

24. Hagerman FC, Walsh SJ, Staron RS, Hikida RS, Gilders RM, Murray TF, et al. Effects of high-intensity resistance training on untraines older men. I. Stength, cardiovascular, and metabolic responses. Gerontol A: Biol Sci Med Sci 2000;55:336-46.

25. Bottaro M, Russo AF, Oliveira RJ. The effects of rest interval on quadriceps torque during an isokinetic testing protocol in elderly. Journal of Sports Science and Medicine 2005;4:285-90.

26. Borg GA. Psychophysical bases of perceived exertion. Med Sci Sports Exerc 1982;14:377-81.

27. Kraemer WJ, Fry AC. Strength testing: Development and evaluation of methodology. In: Maud PJ, Fosters C. Physiological Assessment of Human Fitness. Champaign, IL: Human Kinetics, 1995;115-35.

28. Frontera WR, Meredith CN, O'Reilly KP, Evans WJ. Strength training and determinants of VO2max in older men. J Appl Physiol 1990;68:329-33.

29. Buzzachera CF, Elsangedy HM, Krinski K, Colombo H, Campos W, Silva SG. Efeitos do treinamento de força com pesos livres sobre os componentes da aptidão funcional em mulheres idosas. Revista da Educação Física/UEM 2008;19:195-203.

30. Maiorana AJ, Briffa TG, Goodman C, Hung J. A controlled trial of circuit weight training on aerobic capacity and myocardial oxygen demand in men after coronary artery bypass surgery. J Cardiopulm Rehabil 1997;17:239-47.

31. Hunter GR, Wetzstein CJ, Fields DA, Brown A, Bamman MM. Resistance training increases total energy expenditure and free-living physical activity in older adults. J Appl Physiol 2000;89:977-84.

32. Araújo CGS. Importância de ergoespirometria na prescrição de exercício ao cardiopata. Rev SOCER 1998;11:30-47.

33. McCartney RS, Martin MJ, Sale DG, MacDougall JD. Weight-training-induced attenuation of the circulatory response of older males to weight lifting. N J Appl Physiol 1993;74:1056-60.

34. Shephard RJ. Capítulo 3, IN: Aging, Physical activity, and Health. 1a edição. Human Kinetics, 1997.

35. Izquierdo M, Ibanez J, Hakkinen K, Kraemer WJ, Larrion JL, Gorostiaga EM. Once weekly combined resistance and cardiovascular training in healthy older men. Med Sci Sports Exerc 2004;36:435-43. 